\title{
Research Progress and Prospects of Autophagy in the Mechanism of Multidrug Resistance in Tumors
}

\author{
Wenqing Long $\mathbb{D},{ }^{1}$ Lijun Zhang $\mathbb{D}^{1},{ }^{1}$ Yuxing Wang $\mathbb{D}^{2}$ Haijuan Xie $\mathbb{D}^{2},{ }^{2}$ Lin Wang $\mathbb{D}$, \\ and Hongnu Yu ${ }^{2}{ }^{2}$ \\ ${ }^{1}$ Department of Thoracic Surgery, Affiliated Xinhua Hospital of Dalian University, Dalian 116021, China \\ ${ }^{2}$ Department of Oncology, Affiliated Xinhua Hospital of Dalian University, Dalian 116021, China
}

Correspondence should be addressed to Lin Wang; wanglinbox@sina.com and Hongnu Yu; 879187182@qq.com

Received 9 June 2021; Revised 19 December 2021; Accepted 23 December 2021; Published 30 January 2022

Academic Editor: Dali Zheng

Copyright ( $\odot 2022$ Wenqing Long et al. This is an open access article distributed under the Creative Commons Attribution License, which permits unrestricted use, distribution, and reproduction in any medium, provided the original work is properly cited.

\begin{abstract}
Although the treatment of cancer has made great strides in clinical practice, its high morbidity and fatality rates remain a major threat to human health. Multidrug resistance (MDR) often appears in the process of tumor treatment, leading to tumor refractory and aggravating the risk of tumor recurrence. Therefore, antitumor MDR plays a key role in tumor chemotherapy. Autophagy is an important process for the turnover of intracellular materials, which is commonly seen in the treatment of sensitive and multidrug-resistant tumors, and it can play different roles in various types of MDR tumor cells and tissues. Autophagy plays a dual regulatory role in MDR tumors. On the one hand, autophagy can promote the formation of MDR in tumor cells, weaken the killing effect of chemotherapy drugs on tumor cells, and play a protective role in tumor survival. On the other hand, autophagy production in the cellular environment can kill MDR tumor cells, reverse tumor resistance and enhance the efficiency of chemotherapy drugs. Therefore, the regulation of autophagy to overcome MDR has become increasingly significant in tumor chemotherapy. In this article, we discussed and summarized the research progress of autophagy in MDR tumors, mainly involving the different characteristics of autophagy in MDR cancer cells.
\end{abstract}

\section{Introduction}

Cancer is a major challenge to human health and one of the most important causes of human death among many pathogenic factors. Nowadays, great advances have been made in the clinical treatment of cancer, including radiotherapy, chemotherapy, surgery, molecular targeted therapy, immunotherapy, and so on. There are still many challenges and difficulties in finding a cure for cancer, including cancer recurrence, metastasis, and MDR. These treatment challenges can lead to poor prognosis and high cancer mortality. MDR has become an important factor in the failure of cancer chemotherapy and can lead to recurrence and metastasis. Tumor MDR involves a variety of physiological processes in the cellular environment, including increased drug efflux, decreased uptake of drugs by the body, cell resistance to apoptosis, changed cell cycle points, changed drug effect targets, and eliminated drug toxicity, etc. [1]. In recent years, the molecular mechanisms of MDR have been extensively studied but not fully elucidated.
In normal cells, autophagy can play a role in degrading damaged organelles and misfolded proteins. While removing them, autophagy also participates in the physiological cell metabolism process and makes an important difference in growth and development, adaptation to starvation, cell death, and tumor inhibition $[2,3]$. This prevents the accumulation of excess material in the cell and helps maintain homeostasis. More importantly, this process can release the large molecule materials required for the synthesis of new proteins [4]. In general, phagocytes are regarded as vesicles with scavenging abilities and are the structures that recruit autophagy-related proteins to induce autophagy. In the process of autophagy, the lipid membrane will prolong and form a complete and closed bilayer structure. Eventually, the autophagy lysosome is formed to degrade its encapsulated contents and recover nucleotides, amino acids, and other materials that can be recycled $[5,6]$. Autophagy occurs frequently during tumorigenesis and chemotherapy. In addition, autophagy produced during 
chemotherapy can protect cancer cells from the effects of drug toxicity, leading to tumor refractory and drug resistance [7]. Interestingly, recent research mechanisms suggest that autophagy-related signaling pathways are involved in the occurrence and development of MDR [8]. In recent years, research has explored the methods of using autophagy to reverse MDR tumor cells in the related process of tumor therapy, but the relationship between autophagy and MDR has not been fully studied. Therefore, an in-depth study of the relationship between autophagy and the occurrence and development of MDR tumors and their regulatory mechanisms could provide a new idea for the clinical treatment of tumors. In this paper, the role and treatment of autophagy in MDR tumors are reviewed.

\section{Autophagy}

2.1. Definition of Autophagy and Its Physiological Effects. Autophagy is an ancient cellular decomposition process used to remove excess or dysfunctional organelles and large subcellular structures, thus playing an important housekeeping role in cells. Autophagy is extremely sensitive to nutrient supply and is upregulated at the transcriptional and posttranslational levels in response to nutrient deficiency. It also helps to promote the circulation of cell components and nutrients to maintain the growth and survival of cells [9]. Autophagy plays a beneficial and essential role in the physiological processes of the body, which prevents the formation of toxic and side-effecting protein aggregates and the accumulation of damaged organelles. The accumulation of these substances can lead to cell death, tissue damage, and even the occurrence of chronic inflammation in tumor tissue. Furthermore, autophagy can provide the necessary energy and substrate for intracellular material transport and the survival of an organism $[10,11]$. Autophagy also plays a key role in various tissue processes, immune responses, and the regulation of inflammation [12].

2.2. Discovery of Autophagy-Related Genes. Genetic studies in yeast have laid the foundation for the preliminary discovery of autophagy-related genes and the study of molecular signaling pathways involved in the process of autophagy [13]. So far, more than 40 related genes involved in autophagy formation and regulation have been found. They are essential for coping with microenvironmental stresses such as heat stress, hypoxia, and the accumulation of reactive oxygen species (ROS). And they participate in the induction and initiation of autophagy, the extension of the autophagic membrane, and the mature degradation stages [14].

\subsection{Molecular Mechanisms and Processes of Autophagy.} Because autophagy is a complicated multistep process, mastering the details of autophagy is crucial for researching effective drugs and therapies to regulate autophagy specifically and efficiently. In our understanding, the autophagy process can be separated into the steps in Figure 1.
2.3.1. The ULK1 Complex Participates in the Initiation of Autophagy. The Unc-51-like Kinase 1 (ULK1) complex is composed of an autophagy-related protein 1 (ATG1) homolog from the ULK family (ULK1/2), ATG13, and focal adhesion kinase interacting protein $200 \mathrm{kDa}$ (FIP200). In general, the complex is stable and inactive regardless of nutrient status [15]. However, the association of the mechanistic target of rapamycin complex 1 (mTORC1) with the induction of the complex is influenced by nutrient status.

mTOR is a kind of serine/threonine (Ser/Thr) protein kinase [16], and it plays a significant regulatory role in autophagy. In mammals, mTOR kinase is present in two different complexes, including functional complexes: mTOR complex 1 (mTORC1) and complex 2 (mTORC2) [17]. In mTOR complexes, mTORC1 is more sensitive to rapamycin and can inhibit the initiation of autophagy by phosphorylating the ULK1 complex, while the ULK1 complex is an important forward regulatory factor in the process of autophagosome formation. Under energy-rich circumstances, mTORC1 combines with the ULK1 complex but dissociates under energy deficiency. When mTORC1 associates with the complex, it phosphorylates ULK1/2 and ATG13 to inactivate them. When the energy supply is insufficient, mTORC1 can be separated from the ULK1 complex, thus inducing the formation and extension of the autophagosome membrane and inhibiting cell proliferation (Figure 2).

\subsubsection{The PI3KC3 Complex Is Involved in Nucleation} Regulation. After the ULK1 complex is activated, the class III phosphatidylinositol-3-kinase (PI3KC3) complex is recruited to the presumptive site of autophagosome formation. The PI3KC3 complex consists of PI3K vacuolar protein sorting 34 (Vps34), Vps15, ATG14L, and Beclin1 (BECN1), and it participates in the nucleation of the phagophore.

Some proteins, such as Vps34, autophagy and BECN1 regulator 1 (AMBRA1), and BECN1, have been identified as the ideal regulator proteins in the formation of the phagophore [18-21]. BECN1 is a member of the PI3KC3 complex. The complex is regulated primarily by proteins that mutually interact with BECN1, which is crucial for the complex and autophagy. BECN1 is mainly located in the trans-Golig network (TGN), endoplasmic reticulum, and mitochondria. Its activity is inhibited by the $\mathrm{Bcl}-2$ protein family after binding to Bcl-2, which inhibits autophagy. Under the deficiency of energy, activated c-Jun N-terminal kinase 1 (JNK1) phosphorylates Bcl-2 and interferes with the interaction between $\mathrm{Bcl}-2$ and BECN1. Dissociative BECN1 combines with Vps34 to form a PI3KC3 complex to induce membrane nucleation. In addition, many different mediators, such as ATG14L, AMBRA1 [22, 23], and UV radiation resistance-associated gene (UVRAG) [24], interact with BECN1 to differentially regulate membrane formation. The PI3KC3 complex can phosphorylate phosphatidylinositol to form phosphatidylinositol 3-phosphate (PI3P), and PI3P can mobilize other ATGs in the cytoplasm to bind to the membrane of the proautophagosome, which plays an important role in the early stage of autophagosome formation [25] (Figure 3). 


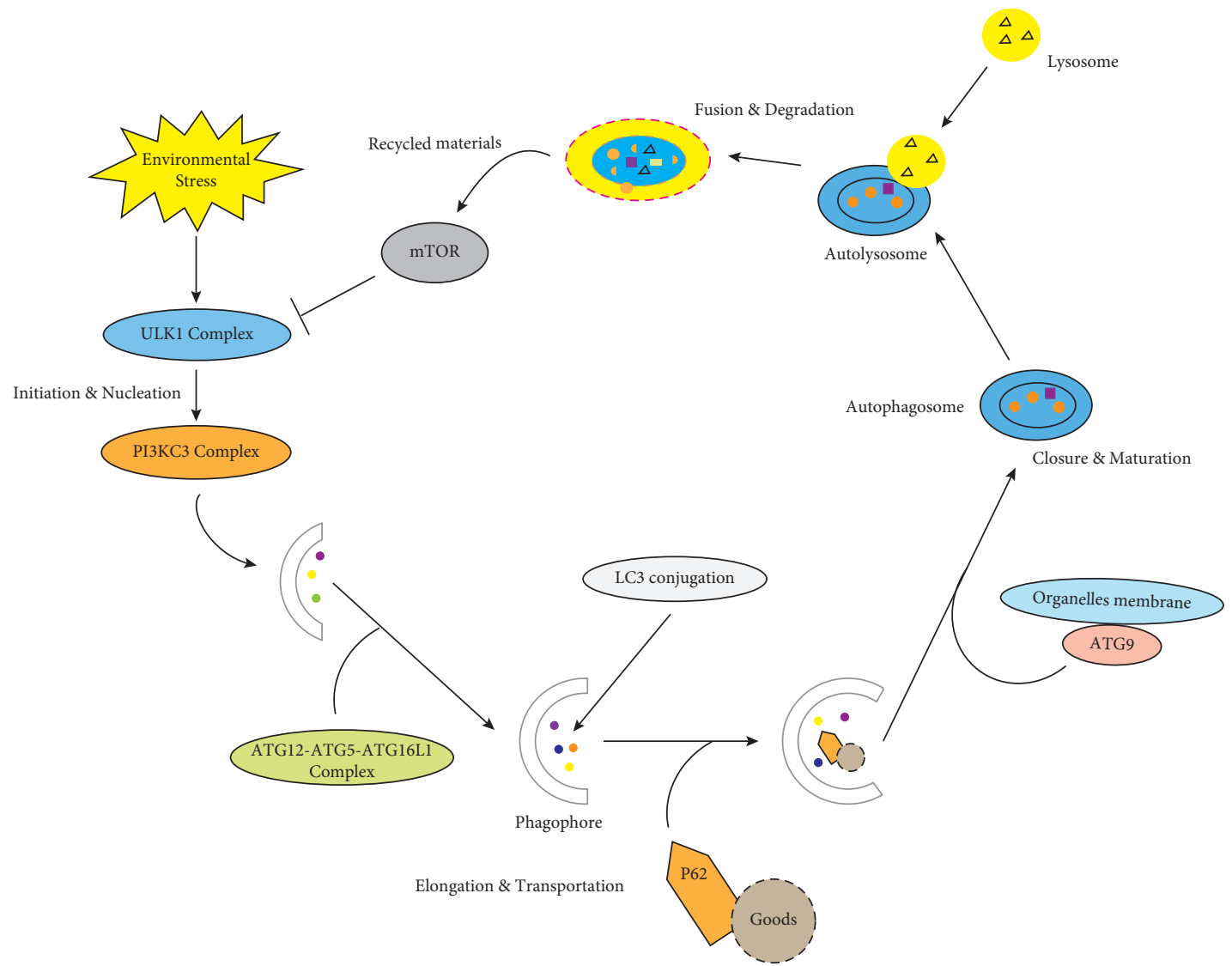

FIGURE 1: A schematic diagram of the autophagy process. This process consists of several phases: Initiation and Nucleation; Elongation and Transportation; Closure and Maturation; Fusion and Degradation. Under the stimulation of external environmental factors such as hypoxia, energy deficiency, and cell stress, etc. The initiation process starts with the activation of the ULK1 complex, and the PI3KC3 complex regulates nucleation. The ATG12-ATG5-ATG16L1 complex and LC3 conjugation participate in the elongation of the phagophore, and the ATG9 protein recruits organelles' membranes to form the autophagic vesicles. After maturation, the autophagosome fuses with the lysosome to form an autolysosome. The autolysosome contents are then degraded.

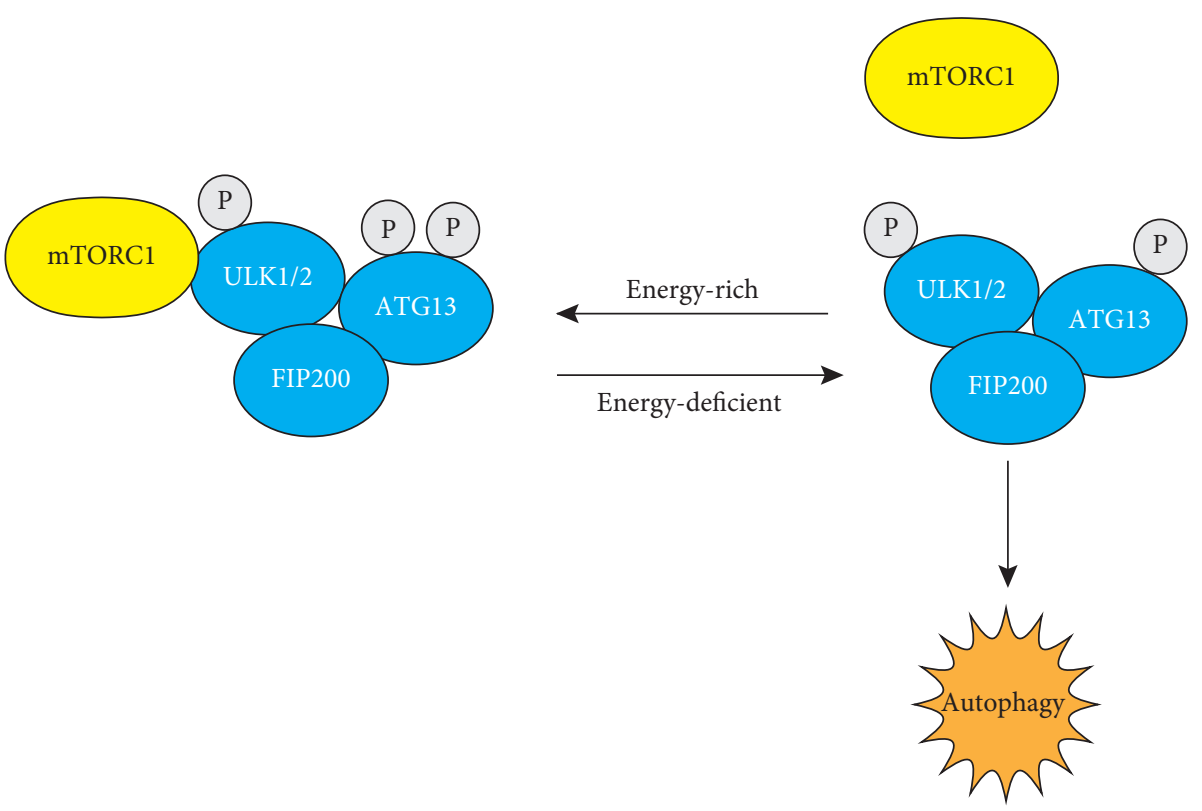

FIGURE 2: A schematic diagram of ULK1 complex induction. Under energy-deficient conditions, mTORC1 dissociates from the ULK1 complex, leaving ULK1/2 and ATG13 partially dephosphorylated, and then the complex can seduce autophagy. Under energy-rich conditions, mTORC1 combines with the complex and inactivates the ULK1/2 and ATG13 proteins across phosphorylation. 


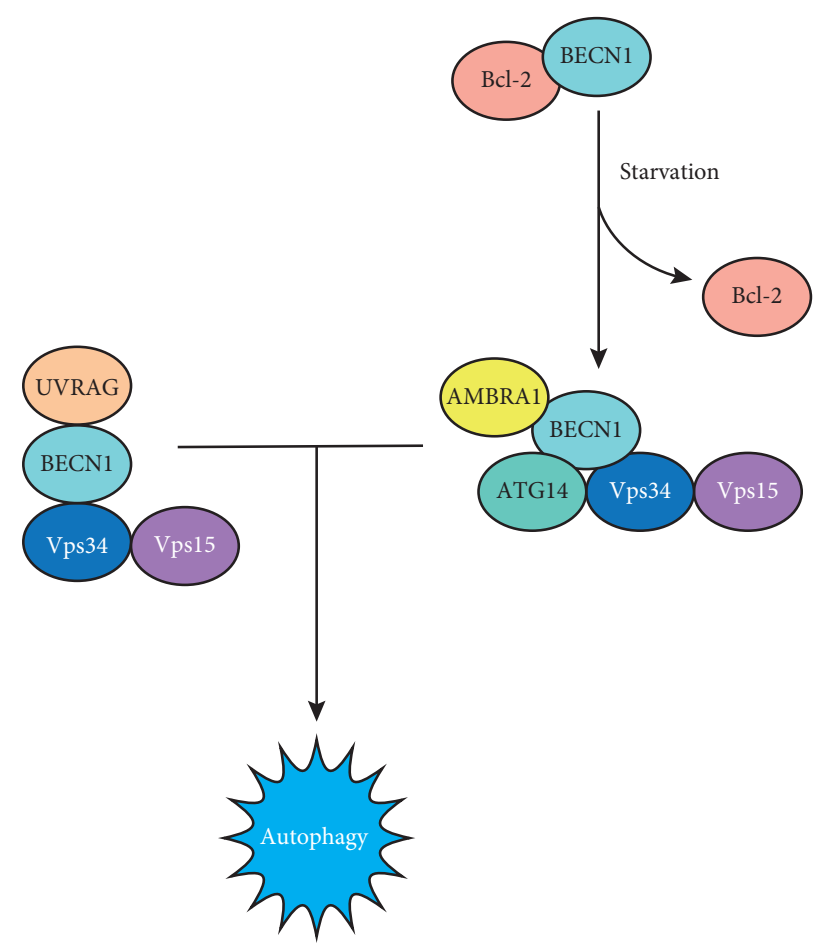

FIgURE 3: A schematic diagram of the PI3KC3 complex involved in the regulation of autophagy. The PI3KC3 complex is necessary for autophagy and is composed of BECN1, ATG14, Vps15, and Vps34. The complex can be positively regulated by AMBRA1 and negatively regulated by Bcl-2, which binds to BECN1 and blocks the combination with the complex. The UVRAG protein is a BECN1 positive mediator, and it can also mediate the activation of the PICKC3 complex to promote autophagy.

2.3.3. The ATG12-ATG5-ATG16L1 Complex and the LC3 Conjugation Cascade. The accumulation of proteins that contain the PI3P binding domain at the membrane nucleation site can lead to the integration of extra ATGs, which is necessary for the expansion and closure of the phagophore membrane. There are two ubiquitin-like (UBL) conjugation systems that can regulate the elongation of the membrane. In the first system, the E1-like enzymes ATG7 and E2-like enzymes ATG10 jointly catalyze the formation of an ATG12-ATG5 conjugated complex [26]. Finally, ATG16L1 interacts with the ATG12-ATG5 complex to form an ATG12-ATG5ATG16L1 complex in a self-oligomerization manner, acting as an E3-like effect for the second UBL coupling system [27-29]. After the autophagy precursor membrane completely fuses to form closed autophagosomes, the ATG12-ATG5-ATG16L1 complex is released into the cytoplasm [30] (Figure 4(a)).

The second UBL system is composed of the conjugation between LC3 and PE. LC3 is involved in the formation of the autophagosome membrane and consists of two interchangeable forms: LC3-I and LC3-II. The intracellular synthesis of LC3 is processed into the cytoplasmic soluble form of LC3-I, during which ATG4 cleaves LC3 to form LC3-I and exposes the C-terminal glycine of LC3 to bind to $\mathrm{PE}$ [31]. PE is conjugated to the C-terminal glycine of LC3-I, and this conjugation needs to be catalyzed by the E1-like enzymes ATG7 and E2-like enzymes ATG3 [32]. Therefore, after processing and modification, LC3-I binds to PE on the membrane surface of the autophagosome and becomes LC3II in the form of membrane binding (Figure 4(b)). LC3-II is located in the proautophagosome and autophagosome and is an important autophagosome marker molecule that increases with autophagosome membrane augmentation [33]. The ratio of LC3-II/LC3-I or the concentration of LC3-II is positively correlated with the number of autophagosomes, reflecting the degree of autophagy activity of cells to a certain extent.

2.3.4. Autophagy Adaptors Transport Autophagic Goods. LC3 can not only act as a sign of the autophagosome membrane, but it can also function as a docking site for a series of goods receptors to bring autophagic goods to autophagic vesicles. Goods receptors such as P62 (also known as sequestosome-1, SQSTM1) bind to LC3 and ubiquitinated substrates, which are subsequently integrated into the autophagosome and degraded in the autophagolysosome [34]. In addition, specific goods receptors preferentially bind to special goods, so goods receptors may provide selectivity for autophagy progress [35].

2.3.5. Maturation of the Autophagosome. After autophagic vesicles form, an additional membrane will be delivered to form the vesicle and close it. The membrane derived from different organelles is recruited to form the autophagic vesicles by ATG9 [36, 37]. It has been proposed that the mammalian homolog of ATG9 shifts localization in 

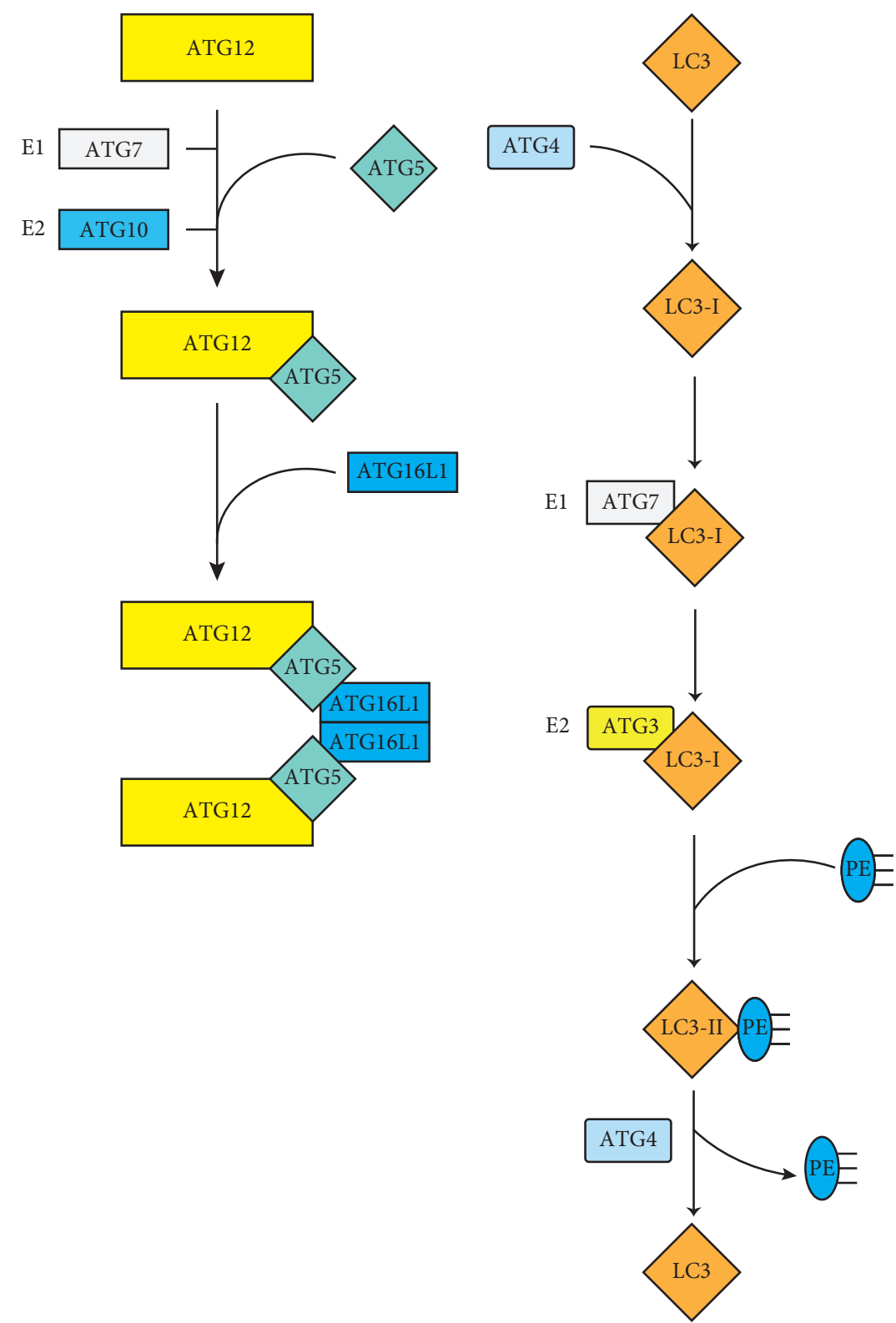

(a)

(b)

Figure 4: (a) The ATG12-ATG5-ATG16L complex. The conjunction of ATG12 and ATG5 begins with the activation of the E1-like enzyme ATG7 and the E2-like enzyme ATG10. Then ATG12-ATG5 combines with ATG16L1 through ATG5. Finally, ATG16L1 dimerizes and binds to the phagosome to promote membrane expansion. (b) The LC3 conjugation system. LC3 is cleaved by ATG4 to form LC3-I and exposes a C-terminal glycine, which can bind to PE. ATG7 is an E1-like enzyme that activates LC3-I and transports it to the E2-like enzyme ATG3. LC3-I is converted to LC3-II by interacting with PE. Eventually, LC3-II can be cleaved by ATG4 to release the PE and LC3.

membrane recruitment [38]. After the isolation membrane completely closes, the vesicle is named an autophagosome.

2.3.6. Fusion of the Autophagosome-Lysosome and Lysosomal Degradation. With the formation of the autophagosome, it will fuse with the lysosome and become an autolysosome. Several proteins, such as VTI1B, syntaxin 17, WAMP8, RAB, and LAMP2, play key roles in the fusion process [39-42].
Finally, the goods are degraded by lysosomal proteases. And degradation products, including amino acids, fatty acids, and nucleotides, are recycled for further use in various kinds of metabolic processes [25].

2.4. Classification of Autophagy. In addition to special lysosomal, ribosomal, and other selective autophagy, according to the types of substrates, modes of transport, and regulatory mechanisms, autophagy can be broadly divided 
into three categories, including microautophagy, macroautophagy, and chaperon-mediated autophagy (CMA) [43-45]. Macroautophagy, commonly referred to as autophagy, is one of the most widely studied types of autophagy and is an ancient and conserved self-degradation process. The main process of its action is that the cytoplasm is surrounded by nonribosomal regions of the endoplasmic reticulum, Golgi apparatus, and other peeling bilayers to remove organelles, pathogens, and protein aggregates and thus play a homeostasis role in normal cells [25]. Microautophagy is a nonselective lysosomal degradation process that involves autophagy tubes directly engulfing cytoplasmic contents on the boundary membrane, mediating invagination and vesicle rupture into the lumen. The main functions of microautophagy are to maintain organelle size, membrane homeostasis, and cell survival under nitrogen deficiency conditions. In addition, microautophagy is coordinated and supplemented with macroautophagy and CMA [46]. Autophagy mediated by CMA is the binding of intracytoplasmic proteins to molecular chaperones and then transporting them to the lysosomal cavity for digestion by lysosomal enzymes. The substrate of CMA is a kind of soluble protein molecule, so the CMA degradation pathway is selective in scavenging proteins, while the former two have no obvious selectivity [47] (Figure 5).

2.5. The Relationship between Autophagy and Tumors. A direct correlation between autophagy and tumors was first discovered in 1999 [48]. The potential role of autophagy in cancer is quite complex and is related to tumor induction and inhibition [12]. Tumor cells are more dependent on autophagy for survival than normal cells, partly because their rapid growth rates change the metabolic and nutrient-deficient growth and living environments. Some chemotherapeutic agents can regulate the process of autophagy, so autophagy-regulated chemotherapy can participate in cancer survival or death $[49,50]$. Abnormal and decreased autophagy restrain the degradation of organelles or proteins in oxidative-stressed cells, resulting in the development of cancer. Besides, autophagy regulation can contribute to the expression of tumor suppressor-associated proteins or oncogenes.Tumor-inhibiting factors are negatively regulated by AMP-activated protein kinase (AMPK) and mammalian target of rapamycin (mTOR), leading to the formation of autophagy and the inhibition of cancer appearance [51]. Based on the dual role of autophagy in tumor genesis and development, inhibition of tumor growth by regulating autophagy activity has gradually become a new research field and direction of autophagy therapy for tumors (Figure 6). To fully understand the process and mechanism of autophagy is of great significance for the clinical treatment of cancer.

\section{Mechanisms of MDR in Tumors}

3.1. Mechanism Categories of MDR. Drug resistance of tumor cells is still one of the biggest obstacles to tumor chemotherapy. It is estimated that about 90 percent of chemotherapy failures are associated with drug-resistant migration and invasion of tumor cells [52]. The mechanisms of multiple drug resistance in tumors can be classified into the following categories: membrane transporters with ATPbinding cassette $(\mathrm{ABC})$ transporters as the main transporters increase drug efflux [53]; DNA repair mechanisms are increased and drug-induced apoptosis is blocked [54]; internal circulation of transports, such as solute carriers, reduces drug absorption [55]; adaptability is enhanced through epigenetic regulation and miRNA regulation [56, 57]; mutations in the P53 pathway or changes in the expression levels of B-cell lymphoma (BCL) family proteins block the transmission of information among apoptosis signaling pathways in intracellular [58,59]; elimination of glutathione S-transferase and cytochrome P450 enzymes promotes drug metabolism [60, 61]; drug target mutations or feedback effects of other targets and signaling pathways block drugmediated tumor toxicity [62]; chemotherapeutic resistance is due to changes in the tumor microenvironment, such as tumor stem cell regulation and hypoxia response [63, 64]; both antiapoptosis of tumor cells and epithelial-mesenchymal transition are participated in tumor drug resistance [65-67]. The mechanisms of cellular drug resistance can be further subdivided into transporter-based classical and nonclassical MDR phenotypes.

3.2. ABC Transporters. The $A B C$ superfamily contains 49 different types of transporters, which can be divided into 7 subfamilies from $A B C-A$ to $A B C-G$ according to sequence similarity and structural composition [68]. Among them, human $\mathrm{ABCB} 1$ is the earliest discovered $\mathrm{ABC}$ transporter. Many studies have confirmed that the overexpression of $\mathrm{ABCB} 1 / \mathrm{MDR} 1$ is the main factor limiting the efficacy of chemotherapy drugs in vitro $[69,70]$. The P-glycoprotein encoded by the MDR1 gene is the most widely studied ABC transporter. P-glycoprotein can use the energy released by ATP decomposition to transport various structurally and functionally unrelated drugs out of cells [71]. In addition to its expression in normal tissues, overexpression of P-glycoprotein can lead to the development of MDR in tumor cells [70]. Therefore, overcoming multiple resistances based on P-glycoprotein has been extensively studied for more than 30 years. $A B C$ transporters are considered to be the main cause of MDR development. The goal of the continued development of antitumor therapy is to block or inactivate $\mathrm{ABC}$ transporters to increase intracellular anticancer drug concentration [72].

\section{The Role of Autophagy in MDR Tumors}

Many literature reports have suggested that autophagy is involved in the process of drug resistance. The research of Bhardwaj et al. [73] found that the expression of ATG5, LC3, and Beclin-1 was significantly increased in tumor tissues and was positively correlated with the expression level of the MDR1 gene. This suggests that autophagy is involved in the occurrence and development of MDR. However, some research evidence has emerged in recent years to show that the role of autophagy in tumorigenesis and development is complex and 

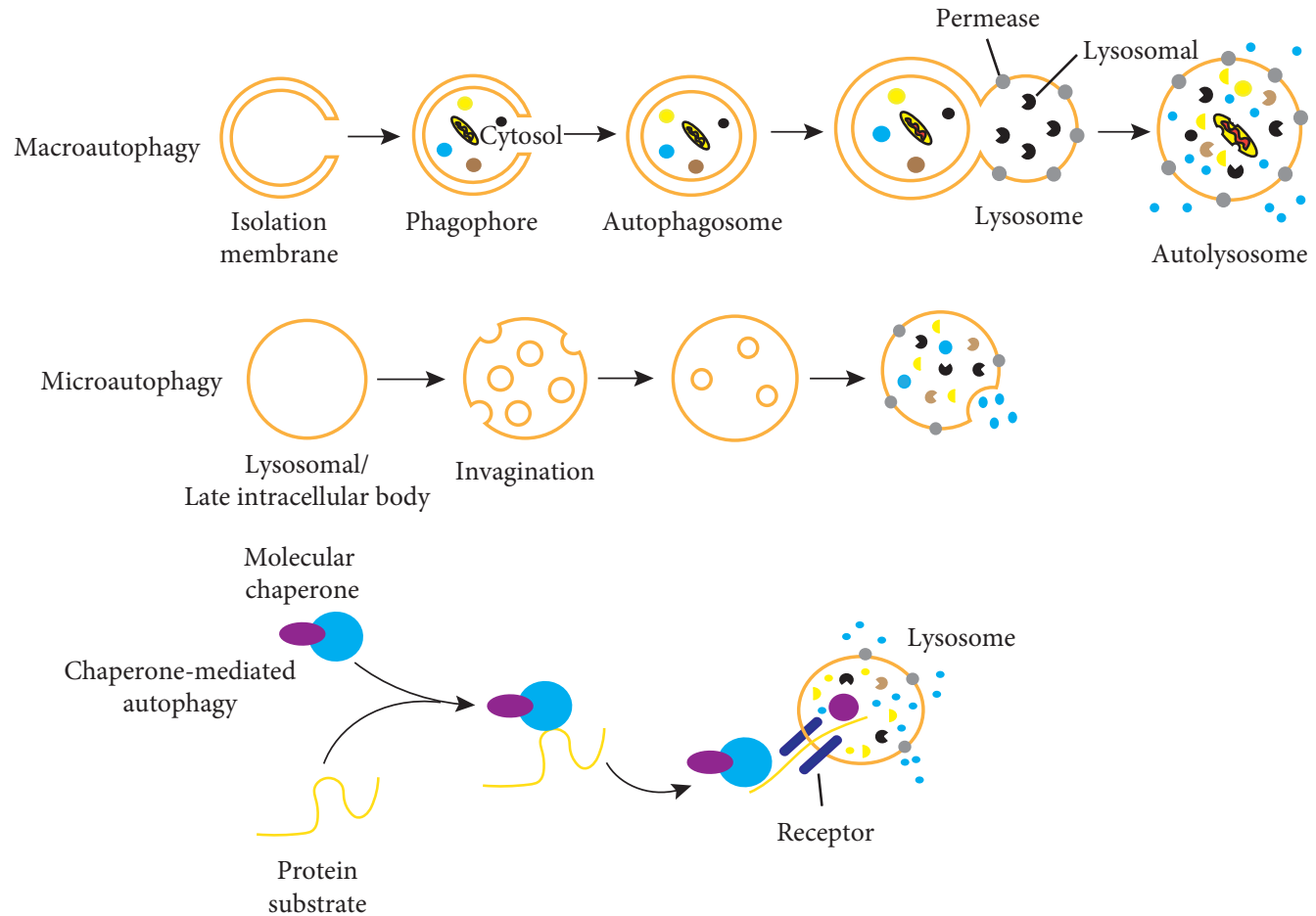

FIGURE 5: Three main types of autophagy. Macroautophagy depends on the formation of cytosolic phagophores to encapsulate and transport contents to the lysosome. Microautophagy absorbs the contents directly through the invagination of the lysosomal membrane. Chaperonemediated autophagy transports the proteins directly across the membrane of the lysosome for digestion.

controversial. Autophagy plays an anticancer role in normal cells by removing damaged organelles, protein components, and circulating products. Paradoxically, excessive autophagy can lead cancer cells to develop "II type programmed cell death" or "autophagic cell death." Autophagy plays a dual role in the occurrence and development of tumors and the resistance of tumor cells to chemotherapy [74]. Autophagy can be activated during antitumor therapy as a protective mechanism for MDR. Therefore, inhibition of autophagy can enhance the sensitivity of tumor cells to chemotherapy and thus enhance the cytotoxicity of chemotherapy drugs. However, autophagy may also induce autophagic cell death. Therefore, autophagy can improve the therapeutic effect of tumor MDR as long as it is properly applied in tumor treatment. The role of autophagy in MDR needs to be clarified.

\subsection{Autophagy Protects MDR Tumors for Survival}

4.1.1. Autophagy Mediates MDR. The ABC transporter is closely related to the occurrence of MDR. Therefore, research advocates the development of drugs that regulate $A B C$ transporters as chemotherapy agents to overcome MDR. However, $\mathrm{ABC}$ transporter modulators have not yet achieved ideal clinical therapeutic effects. In addition, there are many complex phenotypes in MDR. Recent studies have attempted to link autophagy with MDR based on the provided clinical data. In tumor specimens of colorectal cancer patients who survived for 5 years, the expression level of ABCB1 was positively correlated with the expression levels of Beclin1,
LC3, Rictor, and negatively correlated with the expression level of Raptor [75]. It is suggested that autophagy is closely related to the occurrence and development of MDR.

\subsubsection{Autophagy Promotes the Generation and Development} of MDR. Autophagy has been confirmed to promote tumor survival in lung cancer, esophageal cancer, liver cancer, ovarian cancer, kidney cancer, prostate cancer, stomach cancer, pancreatic cancer, breast cancer, colorectal cancer, bladder cancer, and glioma [76-87]. Research has shown that MDR is produced and formed after autophagy. The increased autophagy level in patients with poor prognosis suggests that the presence of autophagy may promote the development of MDR. MDR can be caused by a variety of factors, including the involvement of signaling pathways, various gene targets, and related proteins.

(a) Beclin1 enhances the production of MDR

The scientific research of Lu et al. [88] showed that multiple myeloma (MM) patients with profilin1 (PFN1) expression had a poor prognosis. PFN1 can bind to the Beclin1 complex and promote the initiation of autophagy. The study found that overexpression of PFN1 not only promoted proliferation and bortezomib (BTZ) resistance but also facilitated the process of autophagy and induced BTZ resistance in MM. While inhibition of autophagy by blocking the formation of the Beclin1 complex can reverse BTZ resistance, the results suggest that PFN1 may 


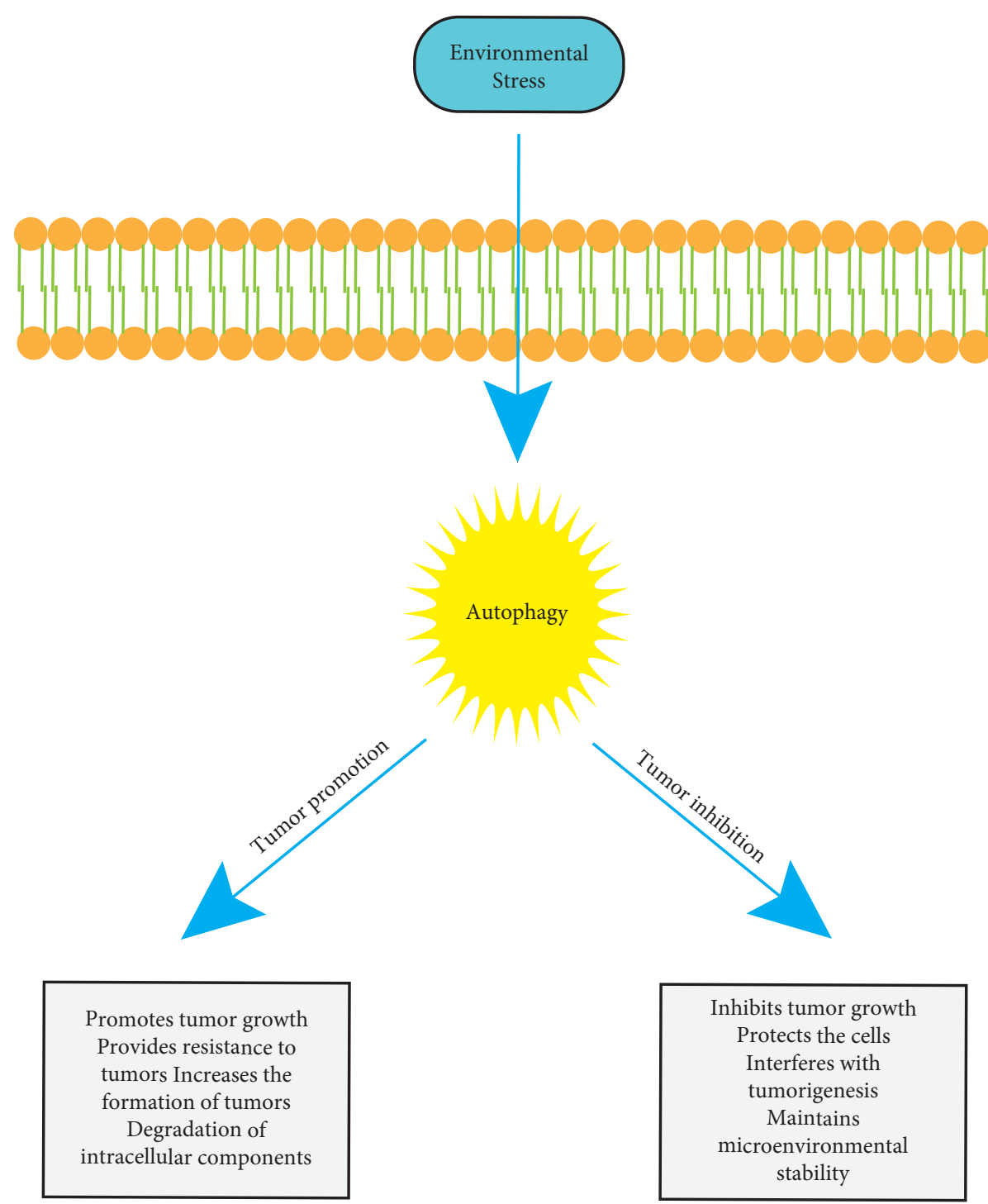

FIGURE 6: A schematic diagram of the autophagy roles of tumor promotion and inhibition in cancer cells.

contribute to BTZ resistance by participating in the Beclin1 complex to promote autophagy. Besides, MDR may be mediated by High-Mobility Group Box 1 (HMGB1). HMGB1 promotes autophagy in response to antitumor drugs. During its transfer from the nucleus to the cytoplasm, by activating the Mitogen-Activated Protein Kinase (MAPK)/Extracellular Signal-Regulated Kinase (ERK) signaling pathway to promote the formation of the Beclin1-class III phosphatidylinositol 3-kinase (Beclin1-PI3KC3) complex to induce autophagy [89]. In addition, HMGB1-induced autophagy participates in the chemoresistance of various kinds of tumors [90].

(b) Both ROS and autophagy contribute to the formation of MDR

In most cases, tumor cells maintain homeostasis and support tumor growth by controlling stromal cell function and activating autophagy [91]. During tumorigenesis, cancer cells induce excessive production of reactive oxygen species (ROS), which activates stromal cells through oxidative stress response mechanisms and autophagy. Both autophagy activation and antioxidant defense mechanisms in the stroma can protect cancer cells from cell damage and death [91]. In addition, the autophagy-mediated matrix is rich in high-energy metabolite cycling, such as L-lactate and ketone bodies, which can support mitochondrial biosynthesis and tumor anabolism [91]. It has been reported that lactate and ketone bodies have the function of chemical inducers for tumor cells and can stimulate the growth and migration of tumor cells. Besides, compared with nonMDR tumor cells and normal cells, the ROS levels and the activity of scavenging/antioxidant enzymes in MDR cancer cells are increased [54, 92]. These results indicate that ROS is closely related to the MDR of tumor cells. 
Autophagy-related MDR has become a challenge in cancer treatment. For example, autophagy promotes the resistance of human lung cancer cells to cisplatin, gefitinib, and erlotinib chemotherapy and facilitates the resistance of oral squamous cell carcinoma to cisplatin [93-96]. Others include temozolomide in glioblastoma; tamoxifen or trastuzumab in breast cancer; 5-fluorouracil (5-FU) in colorectal and esophageal cancer; and leukemia resistance to imatinib treatment [97-102].

\subsubsection{Inhibition of Autophagy Can Boost the Treatment of} MDR Tumors. As a mechanism, autophagy facilitates the formation of resistance to chemotherapy, which weakens the curative effect of anticancer drugs. Thus, inhibition of autophagy may provide a potential tool to enhance the treatment efficiency of tumor cells. Current studies have found many kinds of autophagy suppression methods. Here, we list some of the common autophagy inhibition strategies.

\section{(a) Application of autophagy inhibitors}

In much of the research, the use of autophagy inhibitors can increase the sensitivity of chemotherapeutic agents to tumor cells. Shi and Pan et al. showed that after the inhibition of autophagy by 3 methyladenine (3-MA), a PI3K inhibitor can enhance the cytotoxicity of cisplatin and 5-FU chemotherapeutic agents in nonsmall cell lung cancer $[103,104]$. Another inhibitor, chloroquine (CQ), which blocks the fusion of autophagosomes with lysosomes, also has a similar autophagy inhibition effect. Research has shown that, under in vitro and in vivo conditions, the additional use of CQ enhances the inhibitory effect of 5-FU on the growth of tumor cells [100]. In the research of ovarian cancer SKVCR cells, the application of 3-MA and CQ increased the sensitivity of ovarian cancer MDR cells to chemotherapy by inhibiting autophagy, leading to an increase in cell apoptosis [105]. In human osteosarcoma and melanoma cells, these two inhibitors also increased the sensitivity to apoptosis induced by tumor necrosis factor-related apoptosisinducing ligand (TRAIL) [106]. This inhibition of autophagy has also been shown to be beneficial with the use of other antitumor drugs, such as cisplatin, doxorubicin, and paclitaxel [68].

(b) Inhibition of ATGs

In addition to autophagy inhibitors, inhibition of autophagy by gene silencing of ATG5, Beclin1, and other ATGs can enhance the sensitivity of MDR cells to chemotherapy drugs. For example, in osteosarcoma, miRNA-22 can inhibit the expression of ATG5, Beclin1, and LC3, which can enhance the sensitivity of tumor cells to chemotherapy [107]. In gastric cancer, miR-874 can inhibit autophagy to regulate the MDR of tumor cells by targeting ATG16L1 and enhance the sensitivity of tumor cells to chemotherapy drugs [108]. Autophagy inhibition by targeting ATG12 via small interfering RNAs
(siRNAs) can also enhance the sensitivity of gastric cancer cells to chemotherapy drugs. All the above studies indicate that inhibition of autophagy can improve the utilization efficiency of chemotherapy drugs and reverse tumor MDR to a certain extent.

(c) Regulation of signaling pathways

The efficiency of chemotherapeutic agents can be enhanced by regulating the signaling pathway and the effect of autophagy in MDR tumor cells. Huang et al. revealed the interaction between tyrosine kinase signals coordinated by the HGF-Met axis and autophagy, and the chemotherapy resistance of liver cancer can be reversed by regulating the HGFMet axis and inhibiting autophagy [109]. In their study, Xin et al. [110] found that the inhibition of autophagy in drug-resistant gastric cancer cells by regulating the HULC/FOXM1 signaling pathway could reduce the drug resistance of the cells. In the research of ovarian cancer, targeting microRNA1301 can inhibit the proliferation of cisplatinresistant ovarian cancer cells by inhibiting the NF$\kappa \mathrm{B}$ signaling pathway and regulating autophagy, thus playing a defensive role in the occurrence and development of drug-resistant ovarian cancer cells [111]. All these studies indicate that autophagy and signaling pathways are one of the key factors affecting the chemotherapy of MDR tumor cells while regulating the signaling pathways and inhibiting autophagy are potential strategies to reverse cell MDR.

\subsection{Autophagy Promotes the Death of MDR Tumors}

4.2.1. Autophagy Enhances the Sensitivity of MDR Cells to Chemotherapy. A large number of studies have shown that autophagy is one of the main causes of drug resistance in MDR tumor cells, so it can interfere with the toxic effects of chemotherapy drugs on tumors. It has also been proved that, on the basis of antitumor chemotherapy drugs, increasing autophagy can enhance drug efficacy and thus reverse MDR. For example, nanocrystal of underivatized fullerene C60 (Nano-C60) has cytotoxicity against human hepatoma cells [112]. Harhaji et al. [113] showed that the presence of intracytoplasmic vesicle acidification induced by Nano-60 in glioma cells suggested that autophagy contributed to the cell growth inhibition of Nano-C60. The induction of cell autophagy by Nano-C60 requires photoactivation to enhance the production of ROS. In Adriamycin-resistant MCF-7 breast cancer cell lines, autophagy production induced by Nano-C60 strengthens the chemotherapeutic sensitivity of Adriamycin-resistant tumor cells. Secondly, the chemosensitization effect of Nano-C60 is also dependent on autophagy, because the application of ROS scavenger $\mathrm{N}$-acetyl-L-cysteine not only restrains the autophagy reaction induced by NanoC60 but also significantly inhibits the chemosensitization effect of Nano-C60 [114]. Similarly, Nano-C60 has a variety of biological activities, including induction of 
autophagy and calcium/calmodulin-dependent protein kinase II $\alpha(\mathrm{CaMKII} \alpha)$. CaMKII $\alpha$ is a complex functional protein kinase that can promote the development process of tumor cells. Inhibition of its activity by the chemical inhibitor KN-93 or the knockout of CaMKII $\alpha$ can rapidly facilitate the antitumor activity of Nano-C60. Meanwhile, Nano-C60 can lead to alkalization and enlargement of lysosomes, impair their degradation function, and generate the accumulation of autophagosomes. Excessive accumulation of autophagosomes and inhibition of autophagy degradation play an important role in promoting osteosarcoma cell death. Therefore, inhibition of autophagy degradation can alter and promote the antitumor activity of Nano-C60 [115].

4.2.2. Autophagy Enhances Cell Death in Apoptosis-Deficient $M D R$ Tumors. In apoptosis-deficient MDR tumors, autophagy can generate adaptive responses in vivo that increase the resistance of MDR tumors to chemotherapeutic agents. However, under specific conditions, autophagy can eliminate other influencing factors in the cell apoptosis signaling pathways, making MDR tumors sensitive to apoptosis signals. In ABCB1-overexpressed and etoposideresistant A549 lung cancer cells, interferon A can induce the formation of cell autophagy through the mTOR/ Beclin1/ATG5 pathway. While autophagy inhibition by siRNA silencing Beclin1 can limit the occurrence of interferon A-induced apoptosis, further stimulation of autophagy under the effect of rapamycin can accelerate interferon A-induced apoptosis [116]. In TRAIL drug-resistant prostate cancer, cantharidin-induced production of autophagy can increase TRAIL-mediated apoptosis. Studies have shown that cantharidin could downregulate FADDlike IL-1 $\beta$-converting enzyme (FLICE) inhibitor protein (C-FLIP), upregulate death receptor 5 (DR5), and enhance the level of autophagy, which ultimately strengthens cell apoptosis triggered by TRAIL [117].

\subsubsection{Multiple Factors Promote Autophagy-Mediated MDR Tumor Death}

(a) Drug-mediated autophagy is involved in MDR tumor death

The basic level of autophagy is the mechanism of tumor inhibition by reducing damaged cell parts and proteins and maintaining cell homeostasis [118]. In MDR tumor cells, autophagy can play a prodeath role and trigger autophagic cell death. Some studies are trying to find new antitumor drugs that attempt to kill MDR cells by inducing overautophagy. Kaewpiboon et al. [116] showed that the compound can overcome drug resistance by inducing autophagy, which in turn heightens the process of apoptosis. There is also some research thinking that autophagy helps to remove abnormal and damaged structures or harmful substances from normal cells, while failure to remove these substances can lead to the accumulation of mutations and other cancer-causing substances. Cryptotanshinone and dihydrotanshinone can inhibit the growth of antiapoptotic CRC cells by inducing autophagic cell death and P53-independent cytotoxicity [119]. In a leukemia cell line, drug-resistant cell K562, drug resistance can be antagonized by edifuxin lipid nanoparticles and induced caspaseindependent autophagic cell death [120]. In cisplatinresistant nonsmall cell lung cancer (NSCLC), baicalein can increase cisplatin-induced autophagy to overcome cell resistance and promote drug-resistant cell death [121]. An indole alkaloid drug, Voacamine, can also induce the occurrence of cell autophagy, which mediates the death of MDR tumor cells [122]. In addition, a newly developed drug, Suberoylanilide hydroxamic acid (SAHA), induces autophagic cell death in tamoxifen-resistant breast cancer cells MCF7 and can significantly inhibit the proliferation of tumor cells [123].

(b) The complex role of drug-mediated autophagy on tumor cells

In addition to drug-mediated autophagy-promoting drug-resistant cell death, some ATGs may also be involved in promoting MDR cell death. In antietoposide A549 cells, interferon A not only downregulated the expression of P-glycoprotein but also led to an increase in ATG5, LC3, and Beclin1. These changes are related to the increase in cell apoptosis. Besides, the inhibition of autophagy by taking advantage of siRNA can reduce cell apoptosis, while the level of cell apoptosis can be significantly increased with the addition of an autophagy inducer [116]. However, the failure to increase the level of cell apoptosis while inducing an increase in cell autophagy may also be related to the inhibition of tumor cell proliferation by autophagy. This view was confirmed in the study on the effect of metformin on myeloma cells, namely the expression of autophagy markers was enhanced while the expression of apoptosis-related factors was not found to be boosted when metformin acted on cells [124]. So far, the effects of drugs on tumor cells can be quite different under different conditions, which is still a confusion in many studies. For example, autophagy induced by cisplatin can promote the death of drug-resistant cells in NSCLC, while in oral squamous cell carcinoma, cisplatin-mediated autophagy has the effect of inducing drug resistance and thus protecting tumor cells $[96,121]$. And this phenomenon can be changed by other conditions. Erkisa et al. [125] discovered that the combination of the autophagy inhibitor CQ and the barbiturate palladium (II) complex can enhance the sensitivity of tumor cells to chemotherapeutic drugs and strengthen the apoptosis rate of cells. Furthermore, it should be noted that it is not just chemotherapy drugs that are autophagy inducers; other factors also need to be seriously 
considered in the anticancer treatment process. According to Izdebska et al. [126], the application of lidocaine to rat glioma C6 cell lines can result in the production of protective autophagy.

(c) Signaling pathways participate in promoting the death of MDR tumors

In MDR tumor cells, some signaling pathways may also be involved in autophagic cell death. The combination of sertraline and erlotinib can induce autophagy by regulating the AMPK/mTOR pathway, and the combination of the two drugs can significantly reduce the formation of tumors [127]. Phosphatidylinositol 3-kinase (PI3K) and the mTOR inhibitor NVP-BEZ235 inhibit the proliferation of cisplatin-resistant urothelium carcinoma cells by activating autophagy independent of apoptosis cell death [128]. In lung cancer cells, curcumin-induced autophagy is associated with the activation of the AMPK signaling pathway [129]. In addition, in human melanoma cells, Polygonatum cyrtonema lectin (PCL) induces autophagy through the mitochondriaassociated ROS-p38-p53 pathway [130]. Other cellular signaling pathways may also be involved in the autophagic death of MDR cells. For example, the underlying mechanism of resveratrol-mediated activation of autophagic cell death may be highly dependent on the environment and cell type since it can influence the effect and function of many signaling pathways, including PI3K-AKT, DAPK1, Beclin1, STIM1-mTOR, WNT/ $\beta$-Catenin, and so on [131-136]. On the contrary, in some research, the pathways have a negative regulatory effect on autophagy-promoting MDR cell death. Chen et al. showed that triptolide could increase the chemotherapy sensitivity of A549 cisplatin-resistant cells by inhibiting the $\mathrm{PI} 3 \mathrm{~K} / \mathrm{AKT} / \mathrm{mTOR}$ pathway to induce autophagy in lung cancer cells [137].

Although the exact mechanism of autophagy-mediated MDR cell sensitivity to chemotherapy drugs still needs to be further explored, the findings of the above research highlight the new function of autophagy in the reversal of MDR tumor cell therapy and provide a new plan and strategy for MDR therapy.

\section{Conclusion and Prospects}

Among the multiple treatment methods for tumors, the changes in radiotherapy, chemotherapy, and surgery have made significant progress in the past decades, but MDR is still an urgent problem to be solved in tumor chemotherapy. MDR of tumor cells can significantly inhibit the utilization efficiency of anticancer drugs and plays a key role in the successful treatment of tumors. The generation of MDR can be caused by a variety of factors, which can play an irreplaceable role in the reversal of tumor MDR therapy. The prosurvival and prodeath effects of autophagy may depend on the characteristics of tumor cells and the treatment methods. Autophagy can protect tumor cells to promote survival and can mediate the development of drug resistance in tumor cells during chemotherapy. While the inhibition of autophagy can not only enhance the sensitivity of MDR tumor cells to chemotherapy drugs, in some tumors, autophagy inhibitors can collaborate with chemotherapy drugs to jointly promote the apoptosis of tumor cells. This feature remains mysterious in much of the research on tumors and deserves further exploration. Research has shown that autophagy inhibitors CQ and 3-MA can enhance the cytotoxicity of chemotherapeutic agents when used in combination with some antitumor agents and enhance the sensitivity of MDR tumor cells to drugs. On the other hand, various factors in the cellular environment may affect the production of autophagy. Autophagy inducers mediate the production of autophagy, which can lead to autophagic cell death and eventually kill MDR cells, thus providing a new strategy for anti-MDR cell therapy. Besides, new studies have shown that autophagy can promote MDR cell death, make antiapoptotic MDR cells sensitive to anticancer drugs and reverse tumor cell MDR. This provides an ideal application prospect for autophagy in the treatment of MDR tumors and can overcome the MDR problems of tumor cells with the help of this function of autophagy. Although the molecular mechanism of the interaction between autophagy and tumor MDR has not yet been elucidated, these studies provide clues and solutions for us to further explore the relationship between them and leave a broad space for us to explore.

\section{Conflicts of Interest}

The authors declare that they have no conflicts of interest.

\section{References}

[1] B. C. Baguley, "Multiple drug resistance mechanisms in cancer," Molecular Biotechnology, vol. 46, no. 3, pp. 308-316, 2010.

[2] N. Mizushima, "The pleiotropic role of autophagy: from protein metabolism to bactericide," Cell Death \& Differentiation, vol. 12, no. 2, pp. 1535-1541, 2005.

[3] N. Mizushima, "Autophagy: process and function," Genes \& Development, vol. 21, no. 22, pp. 2861-2873, 2007.

[4] G. Das, B. V. Shravage, and E. H. Baehrecke, "Regulation and function of autophagy during cell survival and cell death," Cold Spring Harbor Perspectives in Biology, vol. 4, no. 6, 2012.

[5] P. Hundeshagen, A. Hamacher-Brady, R. Eils, and N. R. Brady, "Concurrent detection of autolysosome formation and lysosomal degradation by flow cytometry in a high-content screen for inducers of autophagy," $B M C B i$ ology, vol. 9, no. 1, p. 38, 2011.

[6] S. Sridhar, Y. Botbol, F. Macian, and A. M. Cuervo, "Autophagy and disease: always two sides to a problem," The Journal of Pathology, vol. 226, no. 2, pp. 255-273, 2012.

[7] W. J. Buchser, T. C. Laskow, P. J. Pavlik, H.-M. Lin, and M. T. Lotze, "Cell-mediated autophagy promotes cancer cell survival," Cancer Research, vol. 72, no. 12, pp. 2970-2979, 2012.

[8] P. Kumar, D.-M. Zhang, K. Degenhardt, and Z.-S. Chen, "Autophagy and transporter-based multi-drug resistance," Cells, vol. 1, no. 3, pp. 558-575, 2012. 
[9] C. M. Anderson and K. F. Macleod, "Autophagy and cancer cell metabolism," Cellular Nutrient Utilization and Cancer, vol. 347, pp. 145-190, 2019.

[10] A. R. Santiago, L. Bernardino, M. Agudo-Barriuso, and J. Gonçalves, "Microglia in health and disease: a doubleedged sword," Mediators of Inflammation, vol. 2017, Article ID 7034143, 2 pages, 2017.

[11] A. M. K. Choi, S. W. Ryter, and B. Levine, "Autophagy in human health and disease," New England Journal of Medicine, vol. 368, no. 7, pp. 651-662, 2013.

[12] S. Deng, M. K. Shanmugam, A. P. Kumar, C. T. Yap, G. Sethi, and A. Bishayee, "Targeting autophagy using natural compounds for cancer prevention and therapy," Cancer, vol. 125, no. 8, pp. 1228-1246, 2019.

[13] H. Nakatogawa, Y. Ichimura, and Y. Ohsumi, "Atg8, a ubiquitin-like protein required for autophagosome formation, mediates membrane tethering and hemifusion," Cell, vol. 130, no. 1, pp. 165-178, 2007.

[14] Y. Feng, D. He, Z. Yao, and D. J. Klionsky, "The machinery of macroautophagy," Cell Research, vol. 24, no. 1, pp. 24-41, 2014.

[15] C. H. Jung, C. B. Jun, S.-H. Ro et al., "ULK-Atg13-FIP200 complexes mediate mTOR signaling to the autophagy machinery," Molecular Biology of the Cell, vol. 20, no. 7, pp. 1992-2003, 2009.

[16] M. Moloudizargari, M. H. Asghari, E. Ghobadi, M. Fallah, S. Rasouli, and M. Abdollahi, "Autophagy, its mechanisms and regulation: implications in neurodegenerative diseases," Ageing Research Reviews, vol. 40, pp. 64-74, 2017.

[17] G. Kroemer, G. Mariño, and B. Levine, "Autophagy and the integrated stress response,” Molecular Cell, vol. 40, no. 2, pp. 280-293, 2010.

[18] M. Mehrpour, A. Esclatine, I. Beau, and P. Codogno, "Overview of macroautophagy regulation in mammalian cells," Cell Research, vol. 20, no. 7, pp. 748-762, 2010.

[19] J.-M. Park, C. H. Jung, M. Seo et al., "The ULK1 complex mediates MTORC1 signaling to the autophagy initiation machinery via binding and phosphorylating ATG14," Autophagy, vol. 12, no. 3, pp. 547-564, 2016.

[20] K. J. Petherick, O. J. L. Conway, C. Mpamhanga et al., "Pharmacological inhibition of ULK1 kinase blocks mammalian target of rapamycin (mTOR)-dependent autophagy," Journal of Biological Chemistry, vol. 290, no. 18, pp. 1137611383, 2015.

[21] R. C. Russell, Y. Tian, H. Yuan et al., "ULK1 induces autophagy by phosphorylating Beclin-1 and activating VPS34 lipid kinase," Nature Cell Biology, vol. 15, no. 7, pp. 741-750, 2013.

[22] K. Matsunaga, T. Saitoh, K. Tabata et al., "Two Beclin 1binding proteins, Atg14L and Rubicon, reciprocally regulate autophagy at different stages," Nature Cell Biology, vol. 11, no. 4, pp. 385-396, 2009.

[23] F. Strappazzon, M. Vietri-Rudan, S. Campello et al., "Mitochondrial BCL-2 inhibits AMBRA1-induced autophagy," The EMBO Journal, vol. 30, no. 7, pp. 1195-1208, 2011.

[24] C. Liang, P. Feng, B. Ku et al., "Autophagic and tumour suppressor activity of a novel Beclin1-binding protein UVRAG," Nature Cell Biology, vol. 8, no. 7, pp. 688-698, 2006.

[25] E. E. Mowers, M. N. Sharifi, and K. F. Macleod, "Functions of autophagy in the tumor microenvironment and cancer metastasis," FEBS Journal, vol. 285, no. 10, pp. 1751-1766, 2018.
[26] N. Mizushima, T. Noda, T. Yoshimori et al., "A protein conjugation system essential for autophagy," Nature, vol. 395, no. 6700, pp. 395-398, 1998.

[27] N. Mizushima, T. Yoshimori, and Y. Ohsumi, "The role of Atg proteins in autophagosome formation," Annual Review of Cell and Developmental Biology, vol. 27, no. 1, pp. 107-132, 2011.

[28] T. Shpilka, H. Weidberg, S. Pietrokovski, and Z. Elazar, "Atg8: an autophagy-related ubiquitin-like protein family," Genome Biology, vol. 12, no. 7, p. 226, 2011.

[29] K. Tsuboyama, I. Koyama-Honda, Y. Sakamaki, M. Koike, H. Morishita, and N. Mizushima, "The ATG conjugation systems are important for degradation of the inner autophagosomal membrane," Science, vol. 354, no. 6315, pp. 1036-1041, 2016.

[30] A. Kuma, N. Mizushima, N. Ishihara, and Y. Ohsumi, "formation of the $\sim 350-\mathrm{kDa}$ apg12-Apg5-Apg16 multimeric complex, mediated by Apg16 oligomerization, is essential for autophagy in yeast," Journal of Biological Chemistry, vol. 277, no. 21, pp. 18619-18625, 2002.

[31] J. Romanov, M. Walczak, I. Ibiricu et al., "Mechanism and functions of membrane binding by the Atg5-Atg12/Atg16 complex during autophagosome formation," The EMBO Journal, vol. 31, no. 22, pp. 4304-4317, 2012.

[32] Y. Ichimura, T. Kirisako, T. Takao et al., "A ubiquitin-like system mediates protein lipidation," Nature, vol. 408, no. 6811 , pp. $488-492,2000$.

[33] J. Dancourt and T. J. Melia, "Lipidation of the autophagy proteins LC3 and GABARAP is a membrane-curvature dependent process," Autophagy, vol. 10, no. 8, pp. 1470-1471, 2014.

[34] S. Pankiv, T. H. Clausen, T. Lamark et al., "p62/SQSTM1 binds directly to Atg8/LC3 to facilitate degradation of ubiquitinated protein aggregates by autophagy," Journal of Biological Chemistry, vol. 282, no. 33, pp. 24131-24145, 2007.

[35] T. Johansen and T. Lamark, "Selective autophagy: ATG8 family proteins, LIR motifs and cargo receptors," Journal of Molecular Biology, vol. 432, no. 1, pp. 80-103, 2020.

[36] X. Zhuang, K. P. Chung, Y. Cui et al., "ATG9 regulates autophagosome progression from the endoplasmic reticulum in Arabidopsis," Proceedings of the National Academy of Sciences, vol. 114, no. 3, pp. E426-e435, 2017.

[37] S. T. Shibutani and T. Yoshimori, "A current perspective of autophagosome biogenesis," Cell Research, vol. 24, no. 1, pp. 58-68, 2014.

[38] A. R. Young, E. Y. Chan, X. W. Hu et al., "Starvation and ULK1-dependent cycling of mammalian Atg9 between the TGN and endosomes," Journal of Cell Science, vol. 119, no. 18, pp. 3888-3900, 2006.

[39] S. Jäger, C. Bucci, I. Tanida et al., "Role for Rab7 in maturation of late autophagic vacuoles," Journal of Cell Science, vol. 117, no. 20, pp. 4837-4848, 2004.

[40] V. Atlashkin, V. Kreykenbohm, E.-L. Eskelinen, D. Wenzel, A. Fayyazi, and G. Fischer von Mollard, "Deletion of the SNARE vtilb in mice results in the loss of a single SNARE partner, syntaxin 8," Molecular and Cellular Biology, vol. 23, no. 15, pp. 5198-5207, 2003.

[41] E. Itakura, C. Kishi-Itakura, and N. Mizushima, "The hairpin-type tail-anchored SNARE syntaxin 17 targets to autophagosomes for fusion with endosomes/lysosomes," Cell, vol. 151, no. 6, pp. 1256-1269, 2012.

[42] E. L. Eskelinen, "Roles of LAMP-1 and LAMP-2 in lysosome biogenesis and autophagy," Molecular Aspects of Medicine, vol. 27, no. 5-6, pp. 495-502, 2006. 
[43] N. M. Kocaturk, Y. Akkoc, C. Kig, O. Bayraktar, D. Gozuacik, and O. Kutlu, "Autophagy as a molecular target for cancer treatment," European Journal of Pharmaceutical Sciences, vol. 134, pp. 116-137, 2019.

[44] A. S. Patel, D. Morse, and A. M. K. Choi, "Regulation and functional significance of autophagy in respiratory cell biology and disease," American Journal of Respiratory Cell and Molecular Biology, vol. 48, no. 1, pp. 1-9, 2013.

[45] R. Sahu, S. Kaushik, C. C. Clement et al., "Microautophagy of cytosolic proteins by late endosomes," Developmental Cell, vol. 20, no. 1, pp. 131-139, 2011.

[46] W.-W. Li, J. Li, and J.-K. Bao, "Microautophagy: lesserknown self-eating," Cellular and Molecular Life Sciences, vol. 69, no. 7, pp. 1125-1136, 2012.

[47] K. R. Parzych and D. J. Klionsky, "An overview of autophagy: morphology, mechanism, and regulation," Antioxidants and Redox Signaling, vol. 20, no. 3, pp. 460-473, 2014.

[48] X. H. Liang, S. Jackson, M. Seaman et al., "Induction of autophagy and inhibition of tumorigenesis by beclin 1," Nature, vol. 402, no. 6762, pp. 672-676, 1999.

[49] M. T. Rosenfeldt and K. M. Ryan, "The multiple roles of autophagy in cancer," Carcinogenesis, vol. 32, no. 7, pp. 955-963, 2011.

[50] D. A. Gewirtz, "The four faces of autophagy: implications for cancer therapy," Cancer Research, vol. 74, no. 3, pp. 647-651, 2014.

[51] A. Comel, G. Sorrentino, V. Capaci, and G. Del Sal, "The cytoplasmic side of p53's oncosuppressive activities," FEBS Letters, vol. 588, no. 16, pp. 2600-2609, 2014.

[52] T. N. Seyfried and L. C. Huysentruyt, "On the origin of cancer metastasis," Critical Reviews in Oncogenesis, vol. 18, no. 1-2, pp. 43-73, 2013.

[53] S.-Y. Chun, Y.-S. Kwon, K.-S. Nam, and S. Kim, "Lapatinib enhances the cytotoxic effects of doxorubicin in MCF-7 tumorspheres by inhibiting the drug efflux function of ABC transporters," Biomedicine \& Pharmacotherapy, vol. 72, pp. 37-43, 2015.

[54] Q. Cui, J.-Q. Wang, Y. G. Assaraf et al., "Modulating ROS to overcome multidrug resistance in cancer," Drug Resistance Updates, vol. 41, pp. 1-25, 2018.

[55] K. M. Giacomini, K. M. Giacomini, S. M. Huang et al., "Membrane transporters in drug development," Nature Reviews. Drug Discovery, vol. 9, no. 3, pp. 215-236, 2010.

[56] H. Li and B. B. Yang, "Friend or foe: the role of microRNA in chemotherapy resistance," Acta Pharmacologica Sinica, vol. 34, no. 7, pp. 870-879, 2013.

[57] R. H. Shoemaker, "Genetic and epigenetic factors in anticancer drug resistance," JNCI Journal of the National Cancer Institute, vol. 92, no. 1, pp. 4-5, 2000.

[58] A. Bedi, J. Barber, G. Bedi et al., "BCR-ABL-mediated inhibition of apoptosis with delay of $\mathrm{G} 2 / \mathrm{M}$ transition after DNA damage: a mechanism of resistance to multiple anticancer agents," Blood, vol. 86, no. 3, pp. 1148-1158, 1995.

[59] C. S. Wilson, L. J. Medeiros, R. Lai et al., "DNA topoisomerase II $\alpha$ in multiple myeloma: a marker of cell proliferation and not drug resistance," Modern Pathology, vol. 14, no. 9, pp. 886-891, 2001.

[60] G. Filomeni, P. Turella, M. L. Dupuis et al., “6-(7-Nitro-2,1,3benzoxadiazol-4-ylthio)hexanol, a specific glutathione S-transferase inhibitor, overcomes the multidrug resistance (MDR)-associated protein 1-mediated MDR in small cell lung cancer," Molecular Cancer Therapeutics, vol. 7, no. 2, pp. 371-379, 2008.
[61] C. Rodriguez-Antona and M. Ingelman-Sundberg, "Cytochrome P450 pharmacogenetics and cancer," Oncogene, vol. 25, no. 11, pp. 1679-1691, 2006.

[62] D. R. Camidge, W. Pao, and L. V. Sequist, "Acquired resistance to TKIs in solid tumours: learning from lung cancer," Nature Reviews Clinical Oncology, vol. 11, no. 8, pp. 473-481, 2014.

[63] L. Milane, Z. Duan, and M. Amiji, "Role of hypoxia and glycolysis in the development of multi-drug resistance in human tumor cells and the establishment of an orthotopic multi-drug resistant tumor model in nude mice using hypoxic pre-conditioning," Cancer Cell International, vol. 11, no. 1, p. 3, 2011.

[64] M. Dean, T. Fojo, and S. Bates, "Tumour stem cells and drug resistance," Nature Reviews Cancer, vol. 5, no. 4, pp. 275-284, 2005.

[65] F. Azab, S. Vali, J. Abraham et al., "PI3KCA plays a major role in multiple myeloma and its inhibition with BYL719 decreases proliferation, synergizes with other therapies and overcomes stroma-induced resistance," British Journal of Haematology, vol. 165, no. 1, pp. 89-101, 2014.

[66] J. L. Nitiss, "Targeting DNA topoisomerase II in cancer chemotherapy," Nature Reviews Cancer, vol. 9, no. 5, pp. 338-350, 2009.

[67] T. Arumugam, V. Ramachandran, K. F. Fournier et al., "Epithelial to mesenchymal transition contributes to drug resistance in pancreatic cancer," Cancer Research, vol. 69, no. 14, pp. 5820-5828, 2009.

[68] Y.-J. Li, Y.-H. Lei, N. Yao et al., “Autophagy and multidrug resistance in cancer," Chinese Journal of Cancer, vol. 36, no. 1, p. 52, 2017.

[69] R. T. Penson, E. Oliva, S. J. Skates et al., "Expression of multidrug resistance-1 protein inversely correlates with paclitaxel response and survival in ovarian cancer patients: a study in serial samples," Gynecologic Oncology, vol. 93, no. 1, pp. 98-106, 2004.

[70] B. Wang, S. Li, X. Meng, H. Shang, and Y. Guan, "Inhibition of mdr1 by G-quadruplex oligonucleotides and reversal of paclitaxel resistance in human ovarian cancer cells," Tumor Biology, vol. 36, no. 8, pp. 6433-6443, 2015.

[71] H. Glavinas, P. Krajcsi, J. Cserepes, and B. Sarkadi, "The role of $\mathrm{ABC}$ transporters in drug resistance, metabolism and toxicity," Current Drug Delivery, vol. 1, no. 1, pp. 27-42, 2004.

[72] G. N. Zhang, C. R. Ashby, Y. K. Zhang, Z. S. Chen, and $\mathrm{H}$. Guo, " $\beta$ The reversal of antineoplastic drug resistance in cancer cells by $\beta$-elemene," Chinese Journal of Cancer, vol. 34, no. 11, pp. 488-495, 2015.

[73] M. Bhardwaj, H. J. Cho, S. Paul et al., "Vitexin induces apoptosis by suppressing autophagy in multi-drug resistant colorectal cancer cells," Oncotarget, vol. 9, no. 3, pp. 3278-3291, 2018.

[74] R. Mathew, V. Karantza-Wadsworth, and E. White, "Role of autophagy in cancer," Nature Reviews Cancer, vol. 7, no. 12, pp. 961-967, 2007.

[75] W. Shuhua, S. Chenbo, L. Yangyang et al., "Autophagyrelated genes Raptor, Rictor, and Beclin1 expression and relationship with multidrug resistance in colorectal carcinoma," Human Pathology, vol. 46, no. 11, pp. 1752-1759, 2015.

[76] Y. H. Chiu, "Human nonsmall cell lung cancer cells can be sensitized to camptothecin by modulating autophagy," International Journal of Oncology, vol. 53, no. 5, pp. 1967-1979, 2018. 
[77] M. Soltany-Rezaee-Rad, "Overexpression of FOXO3, MYD88, and GAPDH identified by suppression subtractive hybridization in esophageal cancer is associated with autophagy," Gastroenterology Research and Practice, vol. 2014, p. 185035, 2014.

[78] L. Zhan, Y. Zhang, W. Wang et al., "Autophagy as an emerging therapy target for ovarian carcinoma," Oncotarget, vol. 7, no. 50, pp. 83476-83487, 2016.

[79] Q. Zhang, S. Si, S. Schoen, J. Chen, X.-B. Jin, and G. Wu, "Suppression of autophagy enhances preferential toxicity of paclitaxel to folliculin-deficient renal cancer cells," Journal of Experimental \& Clinical Cancer Research, vol. 32, no. 1, p. 99, 2013.

[80] A. M. Blessing, K. Rajapakshe, L. Reddy Bollu et al., "Transcriptional regulation of core autophagy and lysosomal genes by the androgen receptor promotes prostate cancer progression," Autophagy, vol. 13, no. 3, pp. 506-521, 2017.

[81] S. Yang, X. Wang, G. Contino et al., "Pancreatic cancers require autophagy for tumor growth," Genes \& Development, vol. 25, no. 7, pp. 717-729, 2011.

[82] M. W. Han, J. C. Lee, J. Y. Choi et al., "Autophagy inhibition can overcome radioresistance in breast cancer cells through suppression of TAK1 activation," Anticancer Research, vol. 34, no. 3, pp. 1449-1455, 2014.

[83] X. Yang, H. Yin, Y. Zhang et al., " $\alpha$ Hypoxia-induced autophagy promotes gemcitabine resistance in human bladder cancer cells through hypoxia-inducible factor $1 \alpha$ activation," International Journal of Oncology, vol. 53, no. 1, pp. 215-224, 2018.

[84] Y. Niu, X. Yang, Y. Chen et al., "EVI1 induces autophagy to promote drug resistance via regulation of ATG7 expression in leukemia cells," Carcinogenesis, vol. 41, no. 7, pp. 961-971, 2020.

[85] T. Fan, C. Zhang, M. Zong et al., "Peptidylarginine deiminase IV promotes the development of chemoresistance through inducing autophagy in hepatocellular carcinoma," Cell \& Bioscience, vol. 4, no. 1, p. 49, 2014.

[86] W. Yao, P. Guo, Q. Mu, and Y. Wang, "Exosome-derived circ-PVT1 contributes to cisplatin resistance by regulating autophagy, invasion, and apoptosis via miR-30a-5p/YAP1 Axis in gastric cancer cells," Cancer Biotherapy and Radiopharmaceuticals, vol. 36, no. 4, pp. 347-359, 2021.

[87] Y. Fu, L. Hong, J. Xu et al., "Discovery of a small molecule targeting autophagy via ATG4B inhibition and cell death of colorectal cancer cells in vitro and in vivo," Autophagy, vol. 15, no. 2, pp. 295-311, 2019.

[88] Y. Lu, Y. Wang, H. Xu, C. Shi, F. Jin, and W. Li, "Profilin 1 induces drug resistance through Beclin1 complex-mediated autophagy in multiple myeloma," Cancer Science, vol. 109, no. 9, pp. 2706-2716, 2018.

[89] B. Pan, D. Chen, J. Huang et al., "HMGB1-mediated autophagy promotes docetaxel resistance in human lung adenocarcinoma," Molecular Cancer, vol. 13, no. 1, p. 165, 2014.

[90] T. Xu, L. Jiang, and Z. Wang, "The progression of HMGB1induced autophagy in cancer biology," OncoTargets and Therapy, vol. 12, pp. 365-377, 2019.

[91] Z. Zhou, Y. Chen, H. Zhang et al., "Comparison of mesenchymal stromal cells from human bone marrow and adipose tissue for the treatment of spinal cord injury," Cytotherapy, vol. 15, no. 4, pp. 434-448, 2013.

[92] A. Cort, T. Ozben, L. Saso, C. De Luca, and L. Korkina, "Redox control of multidrug resistance and its possible modulation by antioxidants," Oxidative Medicine and Cellular Longevity, vol. 2016, Article ID 4251912, 17 pages, 2016.
[93] D. Yuwen, S. Mi, Y. Ma et al., "Andrographolide enhances cisplatin-mediated anticancer effects in lung cancer cells through blockade of autophagy," Anti-Cancer Drugs, vol. 28, no. 9, pp. 967-976, 2017.

[94] W. Han, H. Pan, Y. Chen et al., "EGFR tyrosine kinase inhibitors activate autophagy as a cytoprotective response in human lung cancer cells," PLoS One, vol. 6, no. 6, p. e18691, 2011.

[95] Y. Zou, Y.-H. Ling, J. Sironi, E. L. Schwartz, R. Perez-Soler, and B. Piperdi, "The autophagy inhibitor chloroquine overcomes the innate resistance of wild-type EGFR nonsmall-cell lung cancer cells to erlotinib," Journal of Thoracic Oncology, vol. 8, no. 6, pp. 693-702, 2013.

[96] S. Li, Y. Wu, Y. Ding, M. Yu, and Z. Ai, "CerS6 regulates cisplatin resistance in oral squamous cell carcinoma by altering mitochondrial fission and autophagy," Journal of Cellular Physiology, vol. 233, no. 12, pp. 9416-9425, 2018.

[97] R. M. Gonçalves, J. P. Agnes, M. Delgobo et al., "Late autophagy inhibitor chloroquine improves efficacy of the histone deacetylase inhibitor SAHA and temozolomide in gliomas," Biochemical Pharmacology, vol. 163, pp. 440-450, 2019.

[98] Y. Wu, Z. Zhang, M. E. Cenciarini et al., “ $\alpha$ Tamoxifen resistance in breast cancer is regulated by the EZH2-er $\alpha$ GREB1 transcriptional Axis," Cancer Research, vol. 78, no. 3, pp. 671-684, 2018.

[99] M. Luque-Cabal, "Mechanisms behind the resistance to trastuzumab in HER2-amplified breast cancer and strategies to overcome it," Clinical Medicine Insights: Oncology, vol. 10, no. 1, pp. 21-30, 2016.

[100] K. Sasaki, N. H. Tsuno, E. Sunami et al., "Chloroquine potentiates the anti-cancer effect of 5-fluorouracil on colon cancer cells," BMC Cancer, vol. 10, no. 1, p. 370, 2010.

[101] T. R. O’Donovan, G. C. O’Sullivan, and S. L. McKenna, "Induction of autophagy by drug-resistant esophageal cancer cells promotes their survival and recovery following treatment with chemotherapeutics," Autophagy, vol. 7, no. 5, pp. 509-524, 2011.

[102] T. Shingu, K. Fujiwara, O. Bögler et al., "Inhibition of autophagy at a late stage enhances imatinib-induced cytotoxicity in human malignant glioma cells," International Journal of Cancer, vol. 124, no. 5, pp. 1060-1071, 2009.

[103] S. Shi, P. Tan, B. Yan et al., "ER stress and autophagy are involved in the apoptosis induced by cisplatin in human lung cancer cells," Oncology Reports, vol. 35, no. 5, pp. 2606-2614, 2016.

[104] X. Pan, X. Zhang, H. Sun, J. Zhang, M. Yan, and H. Zhang, "Autophagy inhibition promotes 5-fluorouraci-induced apoptosis by stimulating ROS formation in human nonsmall cell lung cancer A549 cells," PLoS One, vol. 8, no. 2, p. e56679, 2013.

[105] B. Liang, X. Liu, Y. Liu et al., "Inhibition of autophagy sensitizes MDR-phenotype ovarian cancer SKVCR cells to chemotherapy," Biomedicine \& Pharmacotherapy, vol. 82, pp. 98-105, 2016.

[106] T. Ito, T. Ando, M. Suzuki-Karasaki et al., "Cold PSM, but not TRAIL, triggers autophagic cell death: a therapeutic advantage of PSM over TRAIL," International Journal of Oncology, vol. 53, no. 2, pp. 503-514, 2018.

[107] C. Y. Meng, "MicroRNA-22 regulates autophagy and apoptosis in cisplatin resistance of osteosarcoma," Molecular Medicine Reports, vol. 22, no. 5, pp. 3911-3921, 2020.

[108] H. Huang, J. Tang, L. Zhang, Y. Bu, and X. Zhang, "miR-874 regulates multiple-drug resistance in gastric cancer by 
targeting ATG16L1," International Journal of Oncology, vol. 53, no. 6, pp. 2769-2779, 2018.

[109] X. Huang, G. Gan, X. Wang, T. Xu, and W. Xie, "The HGFMET axis coordinates liver cancer metabolism and autophagy for chemotherapeutic resistance," Autophagy, vol. 15, no. 7, pp. 1258-1279, 2019.

[110] L. Xin, Q. Zhou, Y.-W. Yuan et al., "METase/lncRNA HULC/ FoxM1 reduced cisplatin resistance in gastric cancer by suppressing autophagy," Journal of Cancer Research and Clinical Oncology, vol. 145, no. 10, pp. 2507-2517, 2019.

[111] J. L. Yu and X. Gao, "MicroRNA 1301 inhibits cisplatin resistance in human ovarian cancer cells by regulating EMT and autophagy," European Review for Medical and Pharmacological Sciences, vol. 24, no. 4, pp. 1688-1696, 2020.

[112] C. M. Sayes, A. M. Gobin, K. D. Ausman, J. Mendez, J. L. West, and V. L. Colvin, "Nano-C60 cytotoxicity is due to lipid peroxidation," Biomaterials, vol. 26, no. 36, pp. 7587-7595, 2005.

[113] L. Harhaji, A. Isakovic, N. Raicevic et al., "Multiple mechanisms underlying the anticancer action of nanocrystalline fullerene," European Journal of Pharmacology, vol. 568, no. 1-3, pp. 89-98, 2007.

[114] Q. Zhang, W. Yang, N. Man et al., “Autophagy-mediated chemosensitization in cancer cells by fullerene C60 nanocrystal," Autophagy, vol. 5, no. 8, pp. 1107-1117, 2009.

[115] J. Xu, H. Wang, Y. Hu et al., " $\alpha$ Inhibition of CaMKII $\alpha$ activity enhances antitumor effect of fullerene C60 nanocrystals by suppression of autophagic degradation," Advanced Science, vol. 6, no. 8, p. 1801233, 2019.

[116] C. Kaewpiboon, S. Surapinit, W. Malilas et al., "Feroniellin A-induced autophagy causes apoptosis in multidrug-resistant human A549 lung cancer cells," International Journal of Oncology, vol. 44, no. 4, pp. 1233-1242, 2014.

[117] U. M. Nazim, H. Yin, and S. Y. Park, "Downregulation of c-FLIP and upregulation of DR-5 by cantharidin sensitizes TRAIL-mediated apoptosis in prostate cancer cells via autophagy flux," International Journal of Molecular Medicine, vol. 46, no. 1, pp. 280-288, 2020.

[118] D. Tang, R. Kang, K. M. Livesey et al., "Endogenous HMGB1 regulates autophagy," Journal of Cell Biology, vol. 190, no. 5, pp. 881-892, 2010.

[119] T. Hu, L. Wang, L. Zhang et al., "Sensitivity of apoptosisresistant colon cancer cells to tanshinones is mediated by autophagic cell death and p53-independent cytotoxicity," Phytomedicine, vol. 22, no. 5, pp. 536-544, 2015.

[120] M. A. Aznar, B. Lasa-Saracíbar, and M. J. Blanco-Prieto, "Edelfosine lipid nanoparticles overcome multidrug resistance in K-562 leukemia cells by a caspase-independent mechanism," Molecular Pharmaceutics, vol. 11, no. 8, pp. 2650-2658, 2014.

[121] C.-Y. Sun, Y. Zhu, X.-F. Li et al., "Scutellarin increases cisplatin-induced apoptosis and autophagy to overcome cisplatin resistance in nonsmall cell lung cancer via ERK/p53 and c-met/AKT signaling pathways," Frontiers in Pharmacology, vol. 9, p. 92, 2018.

[122] S. Meschini, M. Condello, A. Calcabrini et al., "The plant alkaloid voacamine induces apoptosis-independent autophagic cell death on both sensitive and multidrug resistant human osteosarcoma cells," Autophagy, vol. 4, no. 8, pp. 1020-1033, 2008.

[123] Y. J. Lee, A. J. Won, J. Lee et al., "Molecular mechanism of SAHA on regulation of autophagic cell death in tamoxifenresistant MCF-7 breast cancer cells," International Journal of Medical Sciences, vol. 9, no. 10, pp. 881-893, 2012.
[124] Y. Wang, W. Xu, Z. Yan et al., "Metformin induces autophagy and G0/G1 phase cell cycle arrest in myeloma by targeting the AMPK/mTORC1 and mTORC2 pathways," Journal of Experimental \& Clinical Cancer Research, vol. 37, no. 1, p. 63, 2018.

[125] M. Erkisa, S. Aydinlik, B. Cevatemre et al., "A promising therapeutic combination for metastatic prostate cancer: chloroquine as autophagy inhibitor and palladium(II) barbiturate complex," Biochimie, vol. 175, pp. 159-172, 2020.

[126] M. Izdebska, M. Hałas-Wiśniewska, W. Zielińska, A. Klimaszewska-Wiśniewska, D. Grzanka, and M. Gagat, "Lidocaine induces protective autophagy in rat C6 glioma cell line," International Journal of Oncology, vol. 54, no. 3, pp. 1099-1111, 2019.

[127] X. Jiang, W. Lu, X. Shen et al., "Repurposing sertraline sensitizes nonsmall cell lung cancer cells to erlotinib by inducing autophagy," JCI Insight, vol. 3, no. 11, 2018.

[128] J.-R. Li, C.-L. Cheng, C.-R. Yang, Y.-C. Ou, M.-J. Wu, and J.-L. Ko, "Dual inhibitor of phosphoinositide 3-kinase/ mammalian target of rapamycin NVP-BEZ235 effectively inhibits cisplatin-resistant urothelial cancer cell growth through autophagic flux," Toxicology Letters, vol. 220, no. 3, pp. 267-276, 2013.

[129] K. Xiao, J. Jiang, C. Guan et al., "Curcumin induces autophagy via activating the AMPK signaling pathway in lung adenocarcinoma cells," Journal of Pharmacological Sciences, vol. 123, no. 2, pp. 102-109, 2013.

[130] B. Liu, Y. Cheng, B. Zhang, H.-J. Bian, and J.-K. Bao, "Polygonatum cyrtonema lectin induces apoptosis and autophagy in human melanoma A375 cells through a mitochondria-mediated ROS-p38-p53 pathway," Cancer Letters, vol. 275, no. 1, pp. 54-60, 2009.

[131] H. Jiang, X. Shang, H. Wu et al., "Resveratrol downregulates $\mathrm{PI} 3 \mathrm{~K} / \mathrm{Akt} / \mathrm{mTOR}$ signaling pathways in human U251 glioma cells," Journal of Experimental Therapeutics \& Oncology, vol. 8, no. 1, pp. 25-33, 2009.

[132] M. S. Choi, Y. Kim, J.-Y. Jung, S. H. Yang, T. R. Lee, and D. W. Shin, "Resveratrol induces autophagy through deathassociated protein kinase 1 (DAPK1) in human dermal fibroblasts under normal culture conditions," Experimental Dermatology, vol. 22, no. 7, pp. 491-494, 2013.

[133] F. Scarlatti, R. Maffei, I. Beau, P. Codogno, and R. Ghidoni, "Role of non-canonical Beclin 1-independent autophagy in cell death induced by resveratrol in human breast cancer cells," Cell Death \& Differentiation, vol. 15, no. 8, pp. 1318-1329, 2008.

[134] F. Scarlatti, R. Maffei, I. Beau, R. Ghidoni, and P. Codogno, "Non-canonical autophagy: an exception or an underestimated form of autophagy?" Autophagy, vol. 4, no. 8, pp. 1083-1085, 2008.

[135] S. Selvaraj, Y. Sun, P. Sukumaran, and B. B. Singh, "Resveratrol activates autophagic cell death in prostate cancer cells via downregulation of STIM1 and the mTOR pathway," Molecular Carcinogenesis, vol. 55, no. 5, pp. 818-831, 2016.

[136] Y. Fu, H. Chang, X. Peng et al., " $\beta$ Resveratrol inhibits breast cancer stem-like cells and induces autophagy via suppressing wnt/ $\beta$-catenin signaling pathway," PLoS One, vol. 9, no. 7, p. e102535, 2014.

[137] L.-M. Chen, T.-J. Song, J.-H. Xiao, Z.-H. Huang, Y. Li, and T.-Y. Lin, "Tripchlorolide induces autophagy in lung cancer cells by inhibiting the PI3K/AKT/mTOR pathway and improves cisplatin sensitivity in A549/DDP cells," Oncotarget, vol. 8, no. 38, pp. 63911-63922, 2017. 